\title{
Appropriateness of antidepressant prescribing: an observational study in a Scottish primary-care setting
}

\author{
Isobel M Cameron, Kenneth Lawton and Ian C Reid
}

\section{ABSTRACT}

\section{Background}

Since the 1990s, Scottish community-based antidepressant prescribing has increased substantially.

Aim

To assess whether GPs prescribe antidepressants appropriately.

\section{Design of study}

Observational study of adults (aged $\geq 16$ years) screened with the Hospital Anxiety and Depression Scale (HADS) attending a GP.

\section{Setting}

Four practices in Grampian, Scotland.

Method

Patients $(n=898)$ completed the HADS, and GPs independently estimated depression status. Notes were scrutinised for evidence of antidepressant use, and the appropriateness of prescribing was assessed.

Results

A total of 237 (26\%) participants had HADS scores indicating 'possible' $(15 \%)$ or 'probable' $(11 \%)$ depression. The proportion of participants rated as depressed by their GP differed significantly by HADS depression subscale scores. Odds ratio for 'possible' versus 'no' depression was 3.54 (95\% confidence interval $[\mathrm{Cl}]=2.17$ to $5.76, P<0.001$ ); and for 'probable' versus 'possible' depression was $3.59(95 \% \mathrm{Cl}=2.06$ to $6.26, P<0.001)$. Similarly, the proportion of participants receiving antidepressants differed significantly by HADS score. Odds ratio for 'possible' versus 'no' depression was $2.79(95 \% \mathrm{Cl}=1.70$ to $4.58, P<0.001)$; and for 'probable' versus 'possible' was $2.12(95 \% \mathrm{Cl}=1.21$ to 3.70, $P=0.009$ ). In 101 participants with 'probable' depression, GPs recognised 53 (52\%) participants as having a clinically significant depression. Inappropriate initiation of antidepressant treatment occurred very infrequently. Prescribing to participants who were not symptomatic was accounted for by the treatment of pain anxiety, or relapse prevention, and for ongoing treatment of previously identified depression.

\section{Conclusion}

There was little evidence of prescribing without relevant indication. Around half of patients with significant symptoms were not identified by their GP as suffering from a depressive disorder: this varied inversely with severity ratings. Rather than prescribing indiscriminately (as has been widely assumed), it is likely that GPs are initiating antidepressant treatment conservatively.

Keywords

antidepressants; depression; primary care.

\section{INTRODUCTION}

Since the 1990s, antidepressant prescribing in Scotland has increased substantially, ${ }^{1}$ as it has elsewhere in the UK. ${ }^{2,3}$

Munoz-Arroyo et al found no increase in the incidence or prevalence of depression, care-seeking behaviour by patients, or identification by GPs to account for the rise in prescription rates. ${ }^{4}$ Suspicion exists, both in the media and in healthcare policy circles, that GPs may be prescribing antidepressants inappropriately. Kendrick et al suggested that GPs' judgements of the severity of depression may be inaccurate, leading to patients with subthreshold symptoms receiving antidepressant prescriptions. ${ }^{5}$ Concern over the rate of antidepressant prescribing has led the Scottish government to set a target to reduce the annual rate of increase of defined daily doses per capita of antidepressant prescribing to zero by $2009 / 2010$, and to 'put in place the required support framework to achieve a $10 \%$ reduction in future years'. ${ }^{6}$

Qualitative studies suggest that GPs' decisions to prescribe for depression are not taken lightly. ${ }^{7,8}$ However, their opinions about their prescribing habits may not reflect actual behaviour.

This study set out to explore prescribing activity as it relates to recognition of depression, documented

IM Cameron, MA Hons Psychology, research fellow; IC Reid, BMedBiol, PhD, FRCPsych, professor of mental health, Department of Mental Health, Clinical Research Centre; K Lawton, FRCGP, GP and clinical senior lecturer, University of Aberdeen, General Practice and Primary Care, Aberdeen.

\section{Address for correspondence}

Isobel M Cameron, University of Aberdeen, Applied Health Sciences (Mental Health), Clinical Research Centre, Royal Cornhill Hospital, Aberdeen AB25 2ZH.

E-mail: i.m.cameron@abdn.ac.uk

Submitted: 2 September 2008; Editor's response: 31 October 2008; final acceptance: 16 March 2009. OBritish Journal of General Practice 2009; 59: 644-649. DOI: 10.3399/bjgp09X454061 
patient history, and current depressive symptoms. It aimed to test the hypothesis that GPs prescribe antidepressants to patients who are unlikely to require them.

\section{METHOD}

\section{Design and setting}

This was an observational study of primary care patients in four practices (one urban, one suburban, and two semi-rural settings) in Grampian, Scotland. Practices were selected with the intention of yielding participants with a mix of socioeconomic status (based on the Scottish Index of Multiple Deprivation [SIMD] 2006 scores). ${ }^{9}$

\section{Participants}

All adult patients (age $\geq 16$ years) were eligible if they were able to understand written and verbal study information and the study questionnaire, all of which assumed an understanding of English.

\section{Procedure for patient participants}

Over five consecutive working days in each practice during September and October 2006, all eligible patients were invited to complete the Hospital Anxiety and Depression Scale (HADS), ${ }^{10}$ along with demographic questions, while waiting to see their GP. Practice receptionists provided information about the study to patients as they checked in. Patients then considered the information in the waiting area. A researcher was present to answer questions and receive consent forms and completed questionnaires. Informed, written consent was obtained. Patients had the option of taking the materials away if they preferred, along with a replypaid envelope. Responses on the HADS depression subscale (HADS-D) were subsequently categorised as 'no' depression for scores <8, 'possible' depression for scores 8-10, and 'probable' depression for scores $\geq 11$, in accordance with established scoring methods.

\section{Procedure for GP participants}

Following the methods of Thompson et al, ${ }^{11}$ after the consultations (and blind to the HADS-D scores), GPs made an estimate of depression status on a four-point scale corresponding to International Statistical Classification of Diseases and Related Problems, 10th revision (ICD-10) severity. ${ }^{12}$ The scale consisted of: no depression (0); mild subclinical emotional disturbance (1); mild clinically important depressive illness (2); and moderate to severe clinically important depressive illness (3). These ratings were dichotomised subsequently between 'clinically important depression' (2 or 3 ) and 'no depression' (0 or 1$)$.

\section{How this fits in}

Antidepressant prescribing increased dramatically during the 1990s in Scotland.

In the absence of evidence to explain this trend, there has been concern that

GPs may be prescribing antidepressants inappropriately. This study found no

evidence to suggest that GPs prescribe antidepressants without relevant

indication. On the contrary, around half of patients with significant symptoms

were not identified by their GP, although this varied by severity. It is likely that

GPs are prescribing conservatively and circumspectly, rather than

indiscriminately.

\section{Case note assessment}

The case notes were reviewed of all patients in the 'possible' and 'probable' depression categories, and in a control sample which included all participants with HADS-D ratings indicating 'no depression' that GPs nonetheless rated as having a clinically important depression. Beyond that, a random sample of controls, stratified by age and sex, to reflect the case sample, was sought. Written recordings were made of documentation of depression history (previous and current) and of the nature of the purpose of consulting on the day of screening. Evidence of current antidepressant prescribing was also noted and assessment made as to whether prescribing was ongoing or newly initiated.

\section{Statistical methods}

Data were analysed using SPSS (version 15.0). Descriptive statistics were produced. Continuous data were assessed for normality using the onesample Kolmogorov-Smirnov test, followed by $t$-test or Mann-Whitney $U$ test as appropriate. Dichotomous data were assessed using $\chi^{2}$ test and binary logistic regression. Odds ratios and $95 \%$ confidence intervals (Cls) are presented. Recognition, antidepressant prescribing, and antidepressant initiation were all investigated according to HADS-D categories

\section{RESULTS}

\section{Participants}

A total 898 of 1475 patients participated (61\%), of whom 897 satisfactorily completed questionnaires for inclusion. A total 813 (90.5\%) questionnaires were completed in the waiting area and 85 (9.5\%) were returned by post. Mean age was 48.8 years (standard deviation [SD] = 18 years); 529 (59\%) participants were female. The median SIMD quintile of home address of participants was 2 (interquartile range $[I Q R]=2-4)$, where 1 represents least deprived and 5 most deprived.

Thirty-three GPs in four practices participated. A further three GPs were on leave during the time of screening. A GP rating was recorded for 889 of the consultations (99\%) of patient participants. 


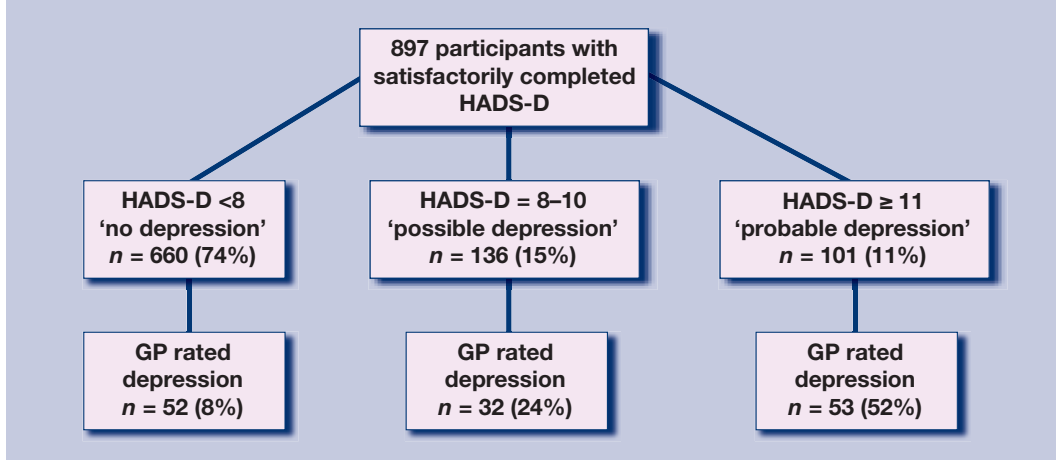

Figure 1. Depression screening and detection rates by Hospital Anxiety and Depression Scale depression subscale (HADS-D) categories.

Figure 2. Hospital Anxiety and Depression Scale depression subscale (HADS-D) score and probability of being rated depressed.

Case notes ( $n=646)$ were reviewed: $232(98 \%)$ notes were available for participants with HADS-D $\geq 8 ; 46$ (88\%) with HADS-D $<8$ that GPs rated as having a clinically important depression; and 368 (61\%) with HADS-D $<8$ that GPs rated as not being depressed. Those with HADS-D $\geq 8$ did not differ significantly from those with HADS-D $<8$ in terms of age (mean 46.5 years, $S D=16.3$ years versus mean 47.8 years, $\mathrm{SD}=17.04$ years; $95 \% \mathrm{Cl}=-1.44$ to 3.95 ) or sex (females $n=141,61 \%$ versus females $n=247,60 \%$; $\chi^{2}=0.077, P=0.42$ ), but differed by SIMD score, with greater deprivation in the 'possible' and 'probable' depression group $($ median $=17.6, \mathrm{IQR}=9.41-26.5$ versus median $=14.04, \mathrm{IQR}=8.92-24.1, P=0.02$ ).

Antidepressants were being prescribed for 45 patients (11\%) with 'no depression'; 34 (25\%) with 'possible depression'; and 41 (42\%) with 'probable depression'. These proportions differed significantly. Odds ratio for 'possible' versus 'no depression' was 2.79 (95\% Cl = 1.70, 4.58, $P<0.001)$; and for 'probable' versus 'possible depression' was $2.12(95 \% \mathrm{Cl}=1.21$, 3.70, $P=0.009$ ). Table 2 breaks down the indications for prescribing in the HADS-D designated 'no depression' group receiving antidepressants.

Thirteen patients had antidepressant treatment initiated during screening. Figure 3 shows that nine of these prescriptions were given for depressive mood symptoms. Two patients had symptoms below threshold, one of whom had a previous history of depression. In line with established guidance, ${ }^{13}$ it is acceptable to initiate antidepressant treatment at a lower threshold to prevent recurrence.

\section{DISCUSSION}

\section{Summary of main findings}

The hypothesis that GPs prescribe antidepressants to patients who are unlikely to require them was not supported. Recent, largely anecdotal, accounts of the

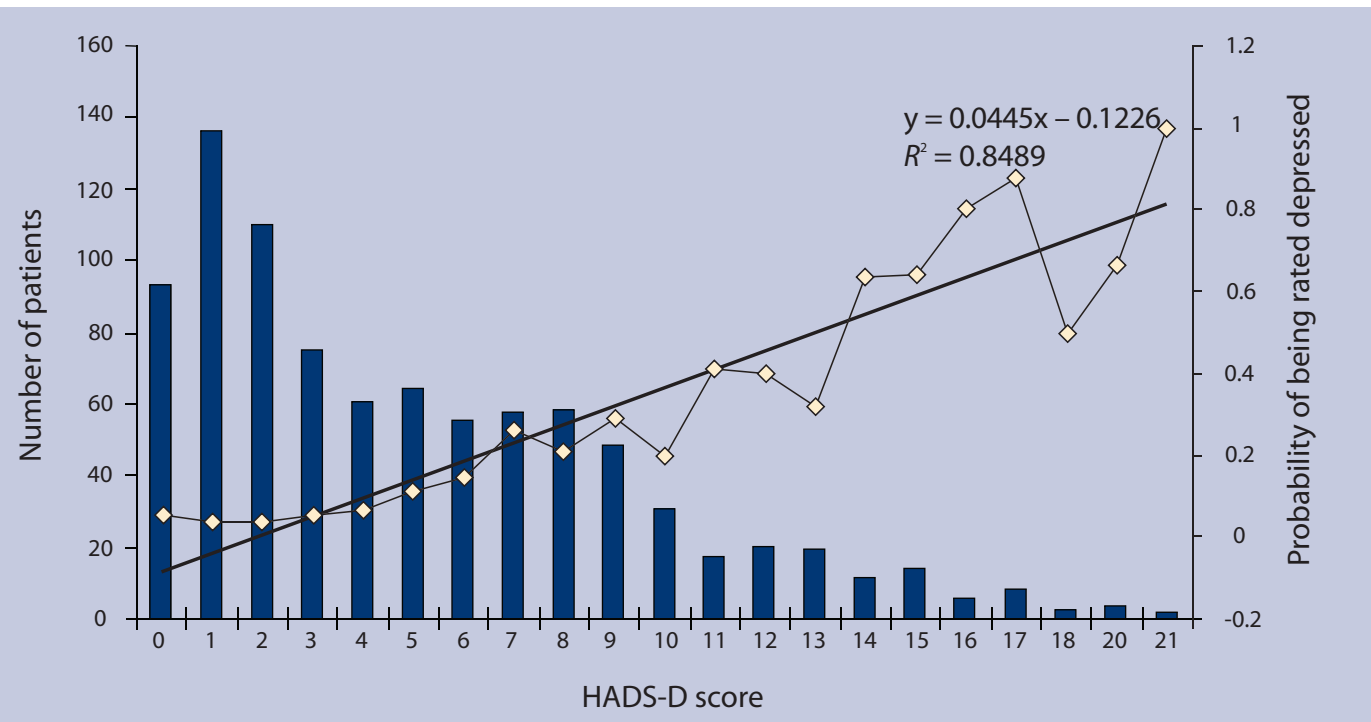


overuse of antidepressants in primary care are unwarranted. Prescribing in those patients who were not symptomatic was accounted for by the treatment of pain, anxiety, or relapse prevention, and for the ongoing successful treatment of previously identified depression. Among patients with 'probable' depression, half did not have their symptomatic status identified and were not offered treatment for depression of any kind, suggesting that GPs may underprescribe, rather than overprescribe antidepressants.

\section{Strengths and limitations of the study}

The majority of eligible patients participated in this study and were broadly representative of adult patients attending Scottish GP surgeries in terms of sex and age. ${ }^{14}$ In terms of deprivation status, the sample was skewed slightly towards a lower level of deprivation than the Scottish average. However, the IQR is the same as for the whole of Scotland. GP participation was exceptionally high.

The use of multiple data sources allowed for a full investigation of mood symptoms and their relationship to GPs' impressions and prescribing behaviour.

The inclusion of participants with depression who were already established on treatment in this sample has allowed for investigation of GPs' clinical impressions relative to the screening tool at stages beyond the outset of treatment. However, it was beyond the scope of this study to assess the appropriateness of prescribing in participants already established on treatment, as it was unknown whether symptoms were of appropriate threshold at the time of treatment initiation. Nonetheless, for such participants, documentation of earlier identified depression was present for over two-thirds, with other indications accounting for almost all of the remainder. By specifically focusing on newly commenced prescribing, it was possible to assess the appropriateness of initiation of treatment, albeit within a small subsample. Further studies are required in larger samples where antidepressant prescribing is newly initiated.

A diagnostic interview, based on ICD-10 criteria, may have measured mood symptoms with greater accuracy than a brief, self-completed measure. However, the HADS has been validated as a screening tool in general practice, ${ }^{15}$ and has demonstrated acceptable sensitivity and specificity in the primary care context. ${ }^{16}$ Asking participants to engage in a full diagnostic assessment would not have been possible in the restricted time allowed prior to GP consultation and would have made participation less acceptable, with a subsequent impact on recruitment.

The findings of this study represent detection of depression during a single visit to primary care.
Table 1. Comparison of demographic characteristics of participants with HADS-D $\geq 8(n=237)$.

\begin{tabular}{lccc} 
Characteristic & $\begin{array}{c}\text { Detected } \\
(n=85)\end{array}$ & $\begin{array}{c}\text { Undetected } \\
(n=152)\end{array}$ & Test \\
\hline Females, $n(\%)$ & $54(64)$ & $90(59)$ & $\chi^{2}=0.43, P=0.51$ \\
\hline Age, mean (SD) & $43.4(14.0)$ & $48.4(17.3)$ & $95 \% \mathrm{Cl}=0.78$ to 9.38 \\
\hline SIMD, median (IQR) & $17.8(9.55$ to 27.5$)$ & $16.9(8.92$ to 25.6$)$ & Mann-Whitney $U$ test $P=0.21$
\end{tabular}

HADS-D $\geq 8=$ Hospital Anxiety and Depression Scale score for the depression subscale indicating 'possible' or 'probable' depression. SIMD = Scottish Index of Multiple Deprivation. $I Q R=$ interquartile range.

\section{Table 2. Antidepressant therapy prescribing to participants} with HADS-D <8.

\begin{tabular}{llc} 
Antidepressant prescribing & Indication & $n=414(\%)$ \\
\hline None & - & $369(89)$ \\
\hline Current & History of depression recorded & $32(8)$ \\
\cline { 2 - 3 } & Anxiety & $5(1)$ \\
\cline { 2 - 3 } & Pain management & $5(1)$ \\
\cline { 2 - 3 } & Unclear & $3(<1)$ \\
\hline
\end{tabular}

HADS-D $\geq 8=$ Hospital Anxiety and Depression Scale score for the depression subscale indicating 'no depression'.

Other investigators have found that over the course of multiple visits, a larger proportion of patients with depression have their depression identified. ${ }^{17}$

\section{Comparison with existing literature}

GP detection rates were similar to those found in previous studies using similar assessment methods, with around one-third of depression being recognised at the 'possible' depression threshold. ${ }^{5,11}$ At the 'probable' depression threshold, approximately half of depression was recognised, consistent with other reports, ${ }^{18,19}$ As described by Thompson et al, ${ }^{20}$ a linear relationship between recognition and HADS-D score
Figure 3. Newly initiated antidepressants, Hospital Anxiety and Depression Scale - depression subscale (HADS-D), and indication. ${ }^{a}$ Both participants consulted with anxiety symptoms and scored 'probable' for anxiety on the HADS anxiety subscale. ${ }^{\circ}$ One participant had a previous history of depression.

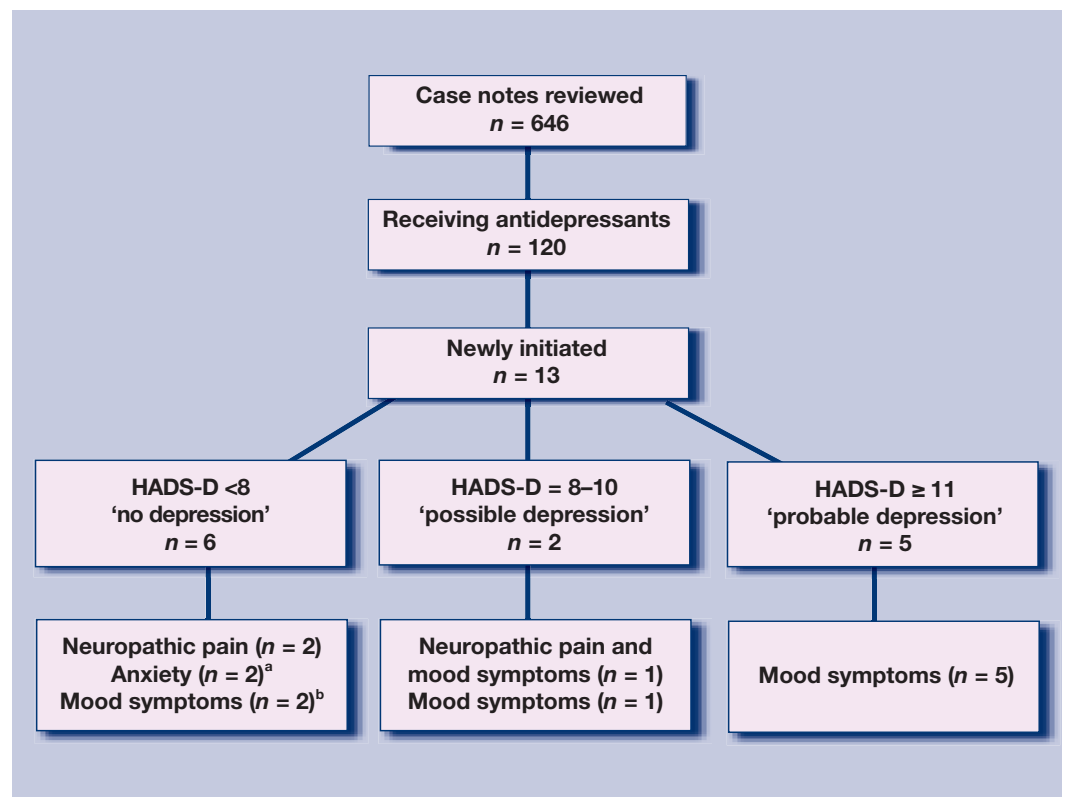


was observed, showing a greater concentration of missed cases at lesser severity. In common with other studies, there was a tendency for greater proportions of false-negative rather than false-positive HADS-D cases. ${ }^{21}$ Crucially, the increases in antidepressant prescribing across Scotland that prompted concern about prescribing behaviour are mirrored in the Grampian area. ${ }^{1}$ These comparisons suggest that the present sample is broadly representative of primary care patients in other parts of the UK. Further, the prescribing behaviour revealed in the sample bears international comparison, in the context of similar increases in antidepressant prescribing. ${ }^{22-24}$ Taken together, these findings confirm that the present sample is representative in terms of population demographics, incidence and severity of mood disorder, and GP activity in terms of detection and prescription for mood disorder.

The very small proportion of patients for whom an antidepressant was newly initiated without obvious indication supports qualitative studies with findings that GPs are cautious in their antidepressant prescribing. ${ }^{7,8}$ The proportion found in the present study is smaller than that found by Kendrick et $a l,{ }^{5}$ where five of 14 patients commenced on antidepressant therapy had HADS-D <8. However, the methods differ in that the current study investigated prescribing in the context of previous history, which showed that prescribing to subthreshold patients was appropriate.

The current study did not reveal GP prescribing to be over inclusive of patients with subthreshold depressive symptoms. Other explanations for increased prescribing in Scotland over the 1990s may relate to an increase in the duration of antidepressant therapy as has recently been reported in England. ${ }^{25}$ Such a finding would represent an improvement in practice, rather than a cause for concern, when compared with prescribing quality in the mid-1990s in Scotland where duration of antidepressant prescribing to individuals was frequently too short to meet established standards. ${ }^{26}$

\section{Implications for future research and clinical practice}

Although the reasons for the increase in prescribing observed since the 1990s remain unclear, this study suggests that the rate may be too low: certainly antidepressants do not appear to be initiated excessively or inappropriately. Concerns have been expressed that target setting to reduce prescribing rates may undermine efforts to reduce suicide rates in Scotland, ${ }^{27}$ although the role of antidepressant drugs in the prevention of suicide remains controversial. ${ }^{28}$ Unfortunately, even efforts to reduce prescribing rates by enhancing the availability of other therapies for depression (such as psychological treatments) may not prove effective for many patients in the face of low recognition rates for mood disorder, particularly those with milder forms of the illness. In any event, it does not appear to be the case that GPs are using chemical antidepressant therapies inappropriately, despite the perceived limited availability of other treatments: if this were so, rates of prescription to patients with subthreshold HADS-D scores would have been much higher than those observed.

The paucity of good-quality evidence relating to the efficacy, effectiveness, and safety of antidepressant drugs and other treatments for depression in primary care, especially with regard to the influence of severity thresholds or symptom duration, limits definitive interpretation of prescribing behaviour. Given the current state of knowledge, it may be concluded that if an error is being committed by GPs, it is likely to be on the side of caution. In the authors' view, efforts should be directed to improving the evidence base to guide the rational deployment of a range of treatments for depressive disorder in primary care, rather than to meet targets of uncertain significance.

\section{Funding body}

Centre for Change and Innovation, Scottish Executive; Support for Science funding, Grampian NHS Research and Development

\section{Ethics committee}

This research was conducted with the approval of the North of Scotland Research Ethics Committee (06/S0802/27)

\section{Competing interests}

Isobel M Cameron has no competing interests to declare. lan C Reid has been paid consultancy and speaker fees by Sanofi Aventis, Wyeth UK, Eli Lilly, and AstraZeneca. Kenneth Lawton has acted as an external primary care consultant for Servier and Pfizer and has been paid speaker fees by Wyeth UK

\section{Acknowledgements}

We would like to thank the primary care participants and general practices who kindly took part: Kirsty Sykes for preparation of research materials; Amanda Cardy of the Scottish Primary Care Research Network (SPCRN) for advice and support on practice recruitment; Graeme Maclennan for statistical advice; and Steve MacGillivray and Tony Kendrick for constructive comment.

\section{Discuss this article}

Contribute and read comments about this article on the Discussion Forum: http://www.rcgp.org.uk/bjgp-discuss

\section{REFERENCES}

1. NHS Quality Improvement Scotland. Clinical indicators report 2007. Glasgow: Quality Improvement Scotland, 2007. http://www.indicators.scot.nhs.uk/Reports/clinical_Indicators_2007.p df (accessed 24 Mar 2009).

2. Hollinghurst S, Kessler D, Peters TJ, Gunnell D. Opportunity cost of antidepressant prescribing in England: analyses of routine data. BMJ 2005; 330(7505): 999-1000.

3. Middleton N, Gunnell D, Whitley E. Secular trends in antidepressant prescribing in the UK, 1975-1998. J Public Health Med 2001; 23(4): 262-267.

4. Munoz-Arroyo R, Sutton M, Morrison J. Exploring potential explanations for the increase in antidepressant prescribing in Scotland using secondary analyses of routine data. Br J Gen Pract 2006; 56(527): 423-428.

5. Kendrick T, King F, Albertella L, Smith P. GP treatment decisions for patients with depression. Br J Gen Pract 2005; 55(513): 280-286. 
6. The Scottish Government. Heat targets: heat target revisions and additions (2008).

http://www.scotland.gov.uk/Topics/Health/health/mental-

health/servicespolicy/DFMH/antidepressantprescribing (accessed 3 Aug 2009).

7. Hyde J, Calnan M, Prior L, et al. A qualitative study exploring how GPs decide to prescribe antidepressants. Br J Gen Pract 2005; 55(519): 755-762.

8. Macdonald S, Morrison JM, Maxwell M, et al. 'A coal face option': GPs' perspectives on the rise in antidepressant prescribing. $\mathrm{Br} J \mathrm{Gen}$ Pract 2009; doi: 10.3399/bigp09X454106.

9. Information Services Division Scotland. General Practice. www.isdscotland.org/isd/3793.html (accessed 3 Aug 2009).

10. Zigmond AS, Snaith P. The hospital anxiety and depression scale. Acto Psychiatr Scand 1983; 67(6): 361-370.

11. Thompson C, Kinmonth AL, Stevens A, et al. Effects of a clinicalpractice guideline and practice-based education on detection and outcome of depression in primary care: Hampshire Depression Project randomised controlled trial. Lancet 2000; 355(9199): $185-191$.

12. World Health Organization. International Statistical Classification of Diseases and Related Health Problems, 10th Revision, Version for 2007. http://www.who.int/classifications/apps/icd/icd10online/ (accessed 24 Mar 2009).

13. National Institute for Clinical Excellence. Depression: management of depression in primary and secondary care. CG23. London: National Institute for Clinical Excellence, 2004.

14. Information Services Division Scotland. General Practice - Practice Team Information. http://www.isdscotland.org/isd/3682.html (accessed 3 Aug 2009).

15. Wilkinson MJB, Barczac P. Psychiatric screening in general practice: comparison of the general health questionnaire and the hospital anxiety and depression scale. J R Coll Gen Pract 1988; 38(312): 311-313.

16. Upadhyaya AK, Stanley I. Hospital anxiety and depression scale. $\mathrm{Br} \mathrm{J}$ Gen Pract 1993; 43(373): 349-350.

17. Rost K, Zhang M, Fortney J, et al. Persistently poor outcomes of undetected major depression in primary care. Gen Hosp Psychiatry 1998; 20(1): 12-20.

18. Dowrick C, Buchan I. Twelve-month outcome of depression in general practice: does detection or disclosure make a difference? BMJ 1995; 311(7015): 1274-1276.

19. Davidson RT, Meltzer-Brody SE. The underrecognition and undertreatment of depression: what is the breadth and depth of the problem? J Clin Psychiatry 1999; 60(Suppl 7): 4-9.

20. Thompson C, Ostler K, Peveler RC, et al. Dimensional perspective on the recognition of depressive symptoms in primary care: the Hampshire Depression Project 3. Br J Psychiatry 2001; 179: 317-323.

21. Cepoiu M, McCusker J, Cole MG, et al. Recognition of depression by non-psychiatric physicians - a systematic literature review and metaanalysis. J Gen Intern Med 2008; 23(1): 25-36.

22. Bellantuono C, Mazzi MA, Tansella M, et al. The identification of depression and the coverage of antidepressant drug prescriptions in Italian general practice. J Affect Disord 2002; 72(1): 53-59.

23. Henriksson S, Asplund R, Boethius G, et al. Infrequent use of antidepressants in depressed individuals (an interview and prescription database study in a defined Swedish population 2001-2002). Eur Psychiatry 2006; 21(6): 355-360.

24. Lecrubier Y. Widespread underrecognition and undertreatment of anxiety and mood disorders: results from three European studies. J Clin Psychiatry 2007; 68(suppl 2): 36-41.

25. Kendrick T, Yuen HM, Dunn N, et al. Why are GPs issuing more and more antidepressant prescriptions year on year? Is depression increasing or are patients being treated for longer? Abstract 12 . Primary Care Research for Better Health: Delivering International Excellence, 4-6 July 2007; The Society for Academic Primary Care, London.

26. MacDonald TM, McMahon AD, Reid IC, et al. Antidepressant drug use in primary care: a record linkage study in Tayside, Scotland. BMJ 1996; 313(7061): 860-861.

27. British Medical Association. Better health, better care: BMA Scotland consultation response.

http://www.bma.org.uk/healthcare policy/responses_consultations/B HBCresponse.jsp?page $=1$ (accessed 3 Aug 2009).

28. Simon G. Antidepressants and suicide. $B M J 2008 ; 336(7643)$ : 515-516. 